NASA/CR—2004-213310

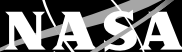

Tests of Mach's Principle With a Mechanical Oscillator

John G. Cramer, Curran W. Fey, and Damon V. Casissi

University of Washington, Seattle, Washington 
Since its founding, NASA has been dedicated to the advancement of aeronautics and space science. The NASA Scientific and Technical Information (STI) Program Office plays a key part in helping NASA maintain this important role.

The NASA STI Program Office is operated by Langley Research Center, the Lead Center for NASA's scientific and technical information. The NASA STI Program Office provides access to the NASA STI Database, the largest collection of aeronautical and space science STI in the world. The Program Office is also NASA's institutional mechanism for disseminating the results of its research and development activities. These results are published by NASA in the NASA STI Report Series, which includes the following report types:

- $\quad$ TECHNICAL PUBLICATION. Reports of completed research or a major significant phase of research that present the results of NASA programs and include extensive data or theoretical analysis. Includes compilations of significant scientific and technical data and information deemed to be of continuing reference value. NASA's counterpart of peerreviewed formal professional papers but has less stringent limitations on manuscript length and extent of graphic presentations.

- TECHNICAL MEMORANDUM. Scientific and technical findings that are preliminary or of specialized interest, e.g., quick release reports, working papers, and bibliographies that contain minimal annotation. Does not contain extensive analysis.

- CONTRACTOR REPORT. Scientific and technical findings by NASA-sponsored contractors and grantees.
- CONFERENCE PUBLICATION. Collected papers from scientific and technical conferences, symposia, seminars, or other meetings sponsored or cosponsored by NASA.

- SPECIAL PUBLICATION. Scientific, technical, or historical information from NASA programs, projects, and missions, often concerned with subjects having substantial public interest.

- TECHNICAL TRANSLATION. Englishlanguage translations of foreign scientific and technical material pertinent to NASA's mission.

Specialized services that complement the STI Program Office's diverse offerings include creating custom thesauri, building customized databases, organizing and publishing research results ... even providing videos.

For more information about the NASA STI Program Office, see the following:

- Access the NASA STI Program Home Page at http://www.sti.nasa.gov

- E-mail your question via the Internet to help@sti.nasa.gov

- Fax your question to the NASA Access Help Desk at 301-621-0134

- Telephone the NASA Access Help Desk at 301-621-0390

- Write to:

NASA Access Help Desk

NASA Center for AeroSpace Information 7121 Standard Drive

Hanover, MD 21076 
NASA/CR—2004-213310

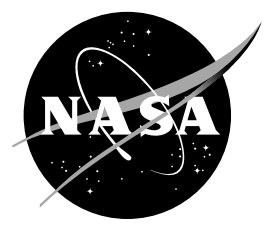

\section{Tests of Mach's Principle With a Mechanical Oscillator}

John G. Cramer, Curran W. Fey, and Damon V. Casissi

University of Washington, Seattle, Washington

Prepared under Contract C-78671-K

National Aeronautics and

Space Administration

Glenn Research Center 


\section{Acknowledgments}

The authors are grateful to Marc G. Millis and the NASA Breakthrough Propulsion Program for providing encouragement and funding for the present experiment. We thank James F. Woodward for providing the very high quality barium titanate capacitors used in the experiment, along with much useful advice.

This report contains preliminary findings, subject to revision as analysis proceeds.

Trade names or manufacturers' names are used in this report for identification only. This usage does not constitute an official endorsement, either expressed or implied, by the National Aeronautics and Space Administration.

Available from

NASA Center for Aerospace Information 7121 Standard Drive

Hanover, MD 21076
National Technical Information Service 5285 Port Royal Road Springfield, VA 22100 


\title{
Tests of Mach's Principle with a Mechanical Oscillator
}

\author{
John G. Cramer ${ }^{\dagger}$, Curran W. Fey, and Damon V. Casissi \\ University of Washington \\ Seattle, Washington 98195-1560
}

\begin{abstract}
James F. Woodward has made a prediction, based on Sciama's formulation of Mach's Principle in the framework of general relativity, that in the presence of an energy flow the inertial mass of an object may undergo sizable variations, changing as the second time derivative of the energy. We describe an attempt to test for the predicted effect with a charging capacitor, using a technique that does not require an unbalanced force or any local violation of Newton's $3^{\text {rd }}$ law of motion. We attempt to observe (1) the gravitational effect of the varying mass and (2) the effect of the mass variation on a driven harmonic oscillator with the charging capacitor as the oscillating mass. We report on the predicted effect, the design and implementation of the measurement apparatus, and initial experience with the apparatus. At this time, however, we will not report on observations of the presence or absence of the Woodward effect.
\end{abstract}

\section{Introduction}

While the principle of equivalence, the exact universal identity of inertial mass and gravitational mass, is a cornerstone of general relativity, our standard model of gravitation, the origins of inertia remain obscure. Mach's Principle, the idea that inertia originates in the gravitational interaction of massive objects with the distant matter of the universe, is an attempt to unify gravitational and inertial mass, but it is not a part of general relativity. Dennis Sciama [1,2] attempted to improve this situation by showing that, for sufficiently symmetric and homogeneous universes, the gravitational interaction of massive objects with distant matter leads to an acceleration-dependent force, i.e., inertia.

James F. Woodward extended Sciama's calculations by introducing an energy flow (e.g., the changing energy in a charging capacitor) into the gravitating system, and demonstrated that the formulation acquires extra transient contributions in Sciama's inertia term that are quite large. The implications of this work are: (a) that it may be possible to modify inertia, and (b) that it may be possible to demonstrate the validity of Mach's Principle with a tabletop experiment.

Woodward and his students [3,4] have attempted to observe the predicted effect by producing an unbalanced force (say, to the right) that is expected to

${ }^{\dagger}$ E-mail address: cramer@phys.washington.edu arise when the inertia varying test mass is accelerated to the right when it has low inertia and to the left when it has high inertia. They report having observed small unbalanced forces, near the limits of their sensitivity, which are about five orders of magnitude smaller than the predicted effect.

Unfortunately, this scheme for observing the inertia variation appears to be at odds with the relativistically invariant form of Newton's 2nd law of motion:

$$
\mathrm{F}=\mathrm{dp} / \mathrm{dt}=\mathrm{mdv} / \mathrm{dt}+\mathrm{vdm} / \mathrm{dt}
$$

Since the inertial mass $\mathbf{m}$ of the test body is expected to vary with time, the last term of equation (1) cannot be ignored. It is not surprising, in view of Newton's 3rd law of motion, that for any sinusoidal variations of the mass around a central value, the force contribution from the $\mathbf{v} \mathbf{d m} / \mathbf{d t}$ term is found to precisely cancel the supposed "unbalanced force" arising from the $\mathbf{m ~ d v / d t ~ t e r m , ~ l e a d i n g ~ t o ~ a ~ t i m e - ~}$ averaged net force of zero on the overall system.

From this simple calculation, it appears that unbalanced force searches are not good tests of the proposed effect. There remains the question of whether the Woodward inertia variation is present in a system having an energy flow. In the present work, we have devised two tests of this effect that do not require the presence of an unbalanced inertial force. 


\section{The Woodward Effect}

Woodward has shown [4] that the relativis-tically invariant wave equation, in the simplest approximation and expressed as a function of an overall scalar gravitational potential $\phi$, has the form:

$$
\begin{aligned}
& \nabla . \phi-\left(1 / c^{2}\right)\left(\partial^{2} \phi / \partial t^{2}\right)=\square \phi=4 \pi G \rho_{0}+ \\
& \left(\phi / \rho_{0} c^{2}\right)\left(\partial^{2} \rho_{0} / \partial t^{2}\right)-\left(\phi / \rho_{0} c^{2}\right)^{2}\left(\partial \rho_{0} / \partial t\right)^{2}
\end{aligned}
$$

where $\boldsymbol{G}$ is Newton's gravitational constant, $\rho_{\mathbf{0}}$ the rest mass density, and $\boldsymbol{c}$ the speed of light. This field equation is obtained only if one assumes, as suggested by Mach's principle, that the local energy density of matter is equal to the matter density times $\phi$. Since Mach's principle demands that $\phi=c^{2}$ when measured locally, this constraint is equivalent to asserting that $E=\boldsymbol{m} \boldsymbol{c}^{2}$.

In writing equation (2), Woodward neglects an additional term of the form $c^{-4}(\partial \phi / \partial t)^{2}$ because it is always small, given its $c^{-4}$ coefficient that is not compensated for by any factor of $\phi$ in the numerator. Combining the last three terms of equation (2) into an effective mass density $\rho(t)$ and solving for this quantity gives the time-dependent effective mass density as:

$$
\begin{aligned}
\rho(t) \approx \rho_{0} & +(1 / 4 \pi G)\left[\left(\phi / \rho_{0} c^{2}\right)\left(\partial^{2} \rho_{0} / \partial t^{2}\right)\right] \\
- & (1 / 4 \pi G)\left[\left(\phi / \rho_{0} c^{2}\right)^{2}\left(\partial \rho_{0} / \partial t\right)^{2}\right] .
\end{aligned}
$$

The second term in equation (3) is $(\mathbf{1} / 4 \pi G)\left[\left(\phi / \rho_{0} c^{2}\right)\left(\partial^{2} \rho_{0} / \partial t^{2}\right)\right]$. This time-dependent fluctuation in the inertial mass can be both positive and negative when $\rho_{0}$ undergoes periodic time variations, e.g., when flow of mass-energy causes time-dependent variations in $\rho_{0}$. This is the inertia-varying term of interest.

In the present work, we will ignore the last timedependent term in equation (3), which has the form $-(\mathbf{1} / 4 \pi G)\left[\left(\phi / \rho_{0} c^{2}\right)^{2}\left(\partial \rho_{0} / \partial t\right)^{2}\right]$. This term is always negative or zero and, for small variations in $\rho_{0}$, will be small compared to the other terms of equation (3).

If a capacitor of capacitance $\mathbf{C}$ is driven by a voltage source that is supplying a time dependent potential $\mathbf{V}(\mathbf{t})=\mathbf{V}_{\mathbf{0}} \operatorname{Sin}(\boldsymbol{\omega} \mathbf{t})$, then the energy in the capacitor, assuming dissipative and inductive effects can be neglected, is $\mathbf{U}(\mathbf{t})=1 / 2 \mathbf{C} \mathbf{V}(\mathbf{t})^{2}=1 / 2 \mathbf{C} \quad \mathbf{V}_{\mathbf{0}}{ }^{2}$ $\operatorname{Sin}^{2}(\mathbf{w t})$. The second time derivative of the energy stored in the capacitor divided by $\mathrm{c}^{2}$ is $\partial^{2} \rho_{0} / \partial t^{2}=$ $\left(\mathbf{1} / \mathbf{c}^{2}\right) \mathbf{d}^{2} U / \mathbf{d t}^{2}=C V_{0}{ }^{2} \omega^{2} \operatorname{Cos}(2 \omega t) / \mathbf{c}^{2}$. This is the $\left(\partial^{2} \rho_{0} / \partial t^{2}\right)$ factor in Woodward's equation (3). The corresponding time dependent variation in inertial mass, assuming that $\phi=\mathbf{c}^{2}$, is then:

$$
\operatorname{dm}(t)=1 /\left(4 \pi \rho_{0} G c^{2}\right) C V_{0}^{2} \omega^{2} \operatorname{Cos}(2 \omega t) .
$$

We will use form for the variation in inertial mass in the analysis that follows. To give this formalism a scale with $\mathrm{c}=2.998 \times 10^{8} \mathrm{~m} / \mathrm{s}$ and $\mathrm{G}=6.672 \times 10^{-11} \mathrm{~m}^{3} / \mathrm{kg}$ $\mathrm{s}^{2}$, let us assume that $\rho_{0}=2,000 \mathrm{~kg} / \mathrm{m}^{3}, \mathrm{C}=9.3 \times 10^{-9} \mathrm{~F}$, $\mathrm{V}_{0}=2,000 \mathrm{~V}$, and $\omega=2 \pi \times 3,400 \mathrm{~Hz}$. With these values:

$$
\operatorname{dm}(t)=0.112 \mathrm{gm} \times \operatorname{Cos}(2 \omega t)
$$

In other words, under these conditions the inertial mass is predicted to vary by about \pm 112 milligrams at twice the capacitor charging frequency, or $6,800 \mathrm{~Hz}$. If the mass of the capacitor and its holder is about $3 \mathrm{gm}$, this would represent a mass variation of about $\pm 3.7 \%$, which should produce quite observable consequences. Note however, that the magnitude of the inertial mass variation is proportional to $\omega^{2}$, and that at lower frequencies it will be reduced accordingly.

It is also useful to consider the maximum current flow that is necessary to charge the capacitor in the manner assumed above, since this is found to place a practical limit on the size of the mass variation. The charge on the capacitor is $\mathbf{q}=\mathbf{C} \mathbf{V}(\mathbf{t})$, so the current is $\mathrm{i}(\mathrm{t})=\mathrm{C} \mathrm{dV} / \mathrm{dt}=\mathrm{C} \mathrm{V}_{0} \omega \operatorname{Cos}(\omega \mathrm{t})=396 \mathrm{~mA} \times \operatorname{Cos}(\omega \mathrm{t})$ for the conditions specified above. It turns out that a high voltage power supply/amplifier capable of delivering a time varying current around $400 \mathrm{~mA}$ at $2 \mathrm{kV}$ represents the most costly component required for the present test of the Woodward effect.

\section{Overview of Two Experimental Tests}

We have undertaken two tests of the Woodward Effect. The first of these tests (T1) depends on the equivalence principle. If an object undergoes a variation in inertial mass, it should also undergo a variation in gravitational mass. In the Earth's gravitational field, it should therefore experience a time-varying gravitational force. If the mass-varying object is part of mechanically resonant system, if the mass variation is at the resonant frequency of the system, and if the gravitational force is in the direction of the oscillation displacement, then the system should excite the oscillator.

Our apparatus, described below, is tested for the presence of such oscillations when the expected gravitational force is (a) in the direction of displacement of the oscillator, and (b) in a direction perpendicular to the direction of displacement of the oscillator. Below we report the results of this test. 
For the second test (T2) we have found, as will be described below, that a mechanical oscillator, driven at resonance, with its inertial mass programmed to vary at the drive frequency, shows sensitive variations in drive-to-response phase and in amplitude, depending on the relative phase of the mass variation and the oscillator drive.

We can test for the presence of the Woodward effect by using the capacitor as the mass in a system that forms a mechanical mass-and-spring oscillator with a resonance frequency of $\mathbf{f}_{\mathbf{0}}$. The oscillator is driven at its resonant frequency, and at the same time, we charge the capacitor at a frequency of $\mathbf{f}_{\mathbf{0}} / \mathbf{2}$, so that in the presence of the Woodward effect the capacitor's inertial mass will vary at frequency $\mathbf{f}_{\mathbf{0}}$.

The effect of the mass variation on the oscillation depends on the relative phase of the mass variation with respect to the oscillator driving force. When it has a relative phase of $\pm \pi / 2$ with respect to the driving force, it causes a positive or negative phase shift in the response motion by shifts of up to a several degrees. On the other hand, if the phase of the mass variation is 0 or $\pi$, it causes an increase or decrease in the amplitude of oscillation.

We have constructed an experiment to attempt to perform both of these tests, but at this writing, only the first test (T1) has been performed, and, as will be described, the results are presently inconclusive.

\section{Experimental Apparatus}

The equipment used in the Mach's Principle test consists of a LabView control and data collection system, a mechanical oscillator system, an electrical driver system for the capacitance, a mechanical driver system for the mechanical oscillator, and a position detection system that measures the oscillator motion.

The LabView signal generator control panel is shown in figure 1. Signal 0 provides the input to the high voltage amplifier that drives the capacitor. Signal 1 provides input to an audio amplifier connected to a voice coil that drives the mechanical oscillator. Signal 0 is selected in frequency $(0-10 \mathrm{kHz})$, amplitude $(0-10 \mathrm{~V})$, voltage offset $( \pm 10 \mathrm{~V})$, and phase $\left(0-360^{\circ}\right)$ by setting panel controls. The frequency of Signal 1 , the oscillator drive signal, is a constant multiplier (usually 2) times the capacitor drive frequency. The system generates sine waves with adjustable phases and amplitudes at two frequencies that differ by a factor of 2 . The amplitude $(0-10 \mathrm{~V})$ and voltage offset $( \pm 10 \mathrm{~V})$ of Signal 1 are set by setting panel controls.
The phase of Signal 1 is set to that of Signal 0 with the phase control set to 0 .

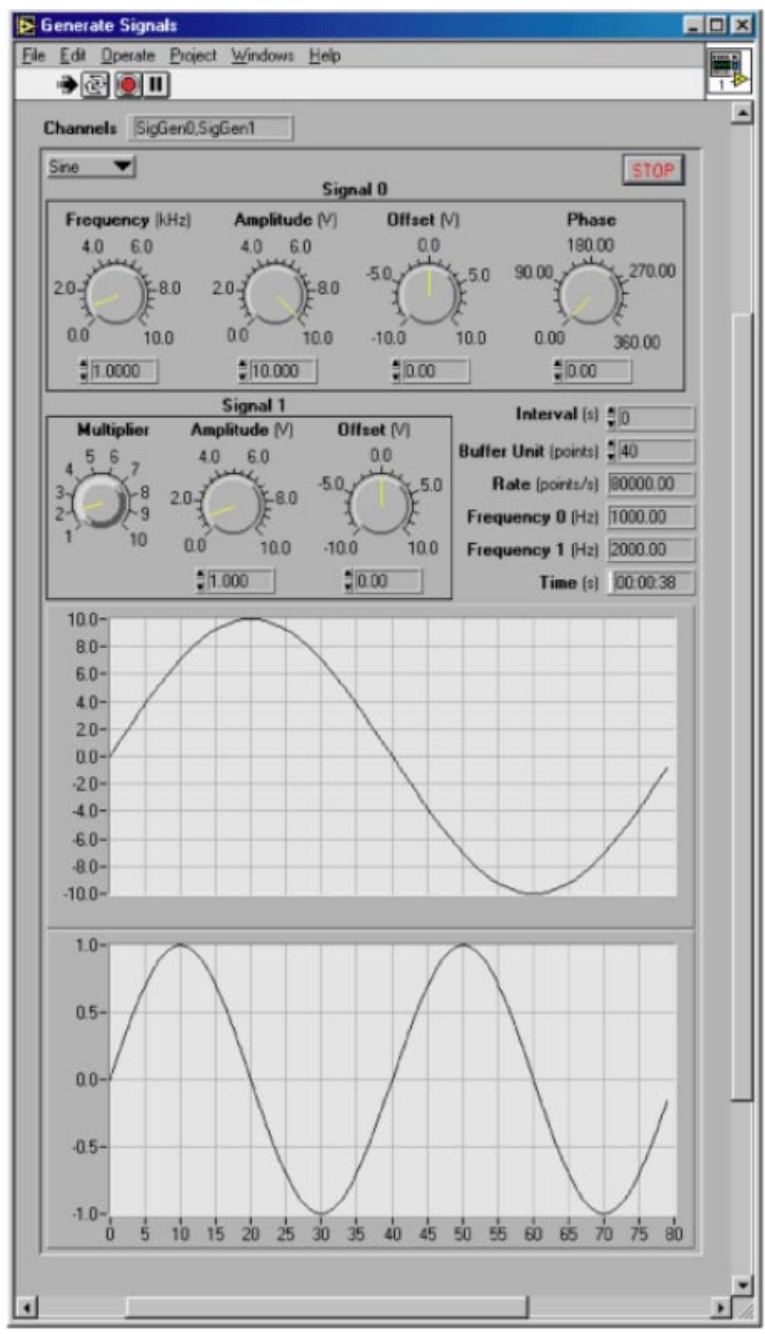

Figure 1 LabView Signal Generation Control Panel

The display windows of the signal generator control panel show the two generated signals and their relative phases.

Figure 2 shows the LabView panel for the data acquisition system. The system samples the mechanical drive voltage and the position measurement of the oscillator, as separate data streams recorded on the system hard disk. In the present configuration, these streams are read back in at the conclusion of a data run and analyzed to extract the relative phase of the drive and response signals by integration. 


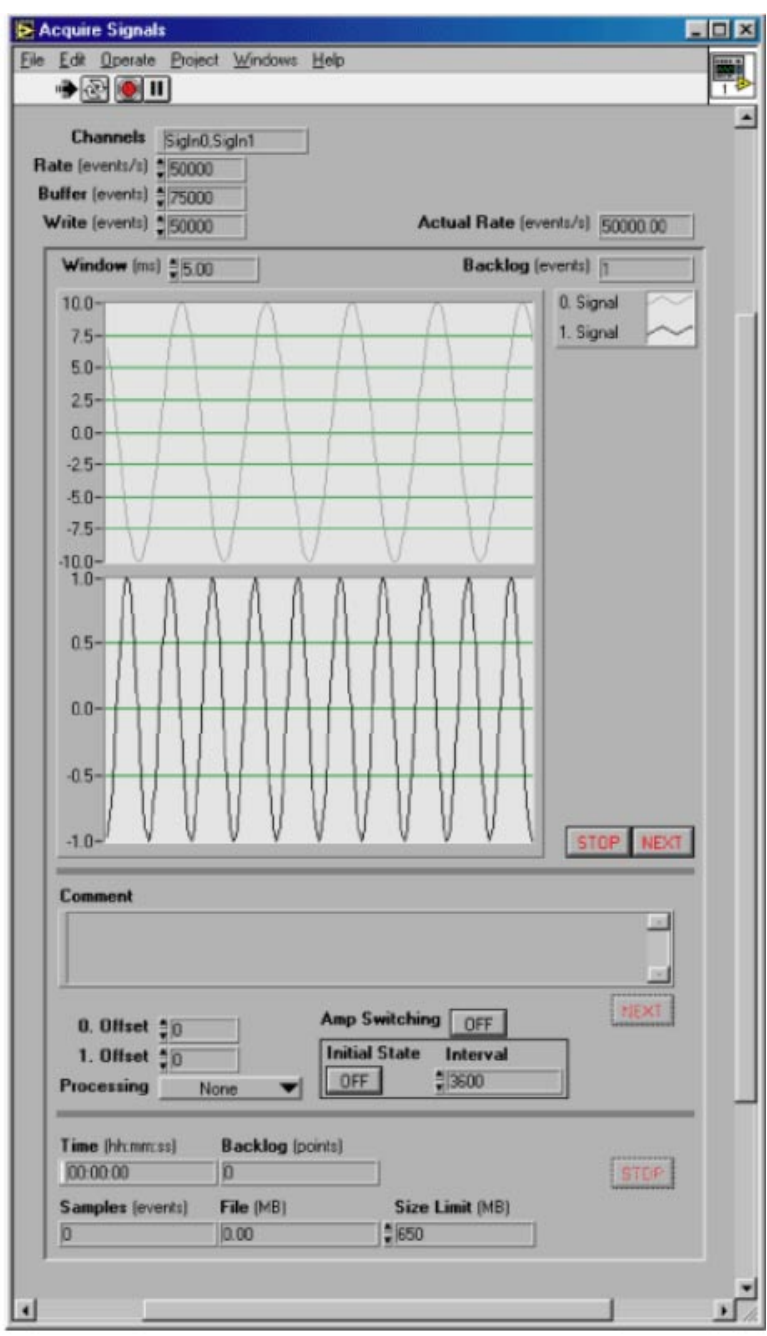

Figure 2 LabView Data Acquisition Control Panel

The "Mach Guitar" mechanical oscillator system is shown in figure 3. A capacitor is suspended between two strings of 13 gauge steel music wire. The tensioning mechanisms for the strings, adapted from musical stringed instrument tensioning modules, is shown in the lower part of the diagram and the rotati insulated tension equalizer is shown at the top of the diagram. The strings are electrically isolated from eac other and from the frame, so that a driving potenti ng up to $3 \mathrm{kV}$ can be applied between them for chargi the capacitor. Figure 3 also shows the support structures for the sensor (horizontal bar at center) and for the voice-coil driver (double-barred $\mathrm{H}$ structure).

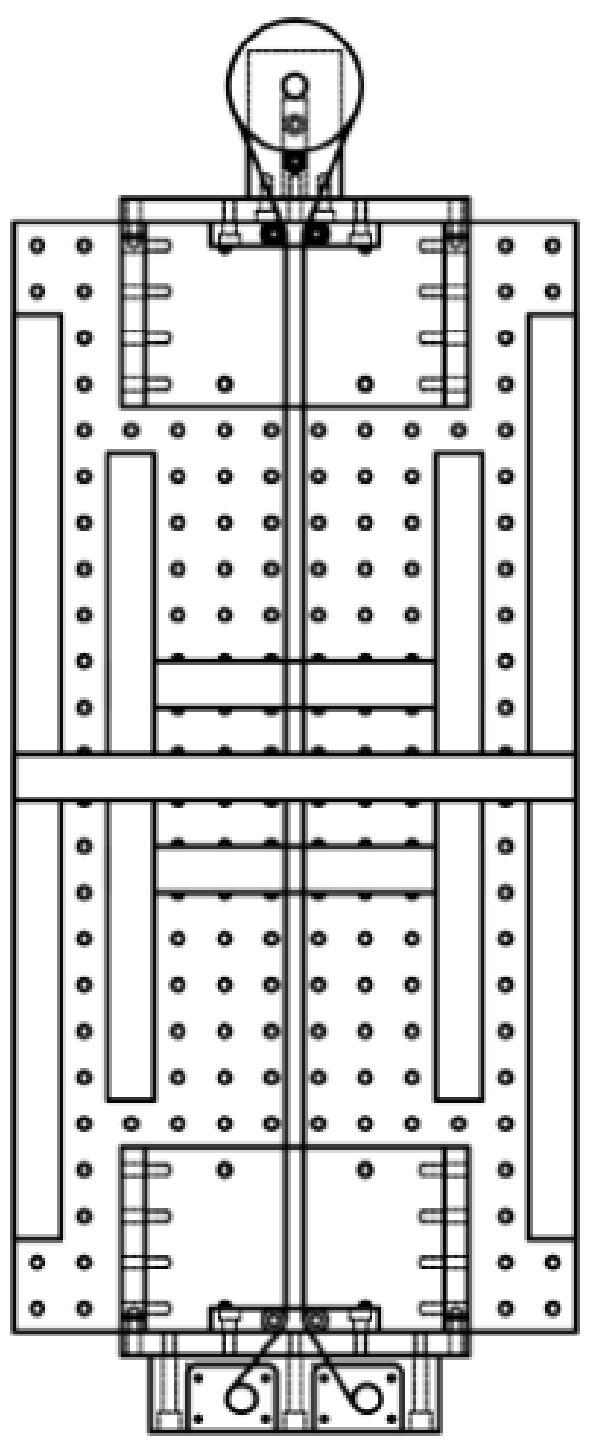

Figure 3 Top view of the "Mach Guitar" Mechanical Oscillator system

Figure 4 shows a side view of the Mach Guitar mechanical oscillator system. The support for the position measuring sensor is shown in the top part of the figure, and the support for the voice coil oscillator drive is shown below. The strings are shown across the mid line of the diagram, with the string tensioning mechanism on the left and the tension equalizer on the right. The direction of resonant oscillation is vertical. 


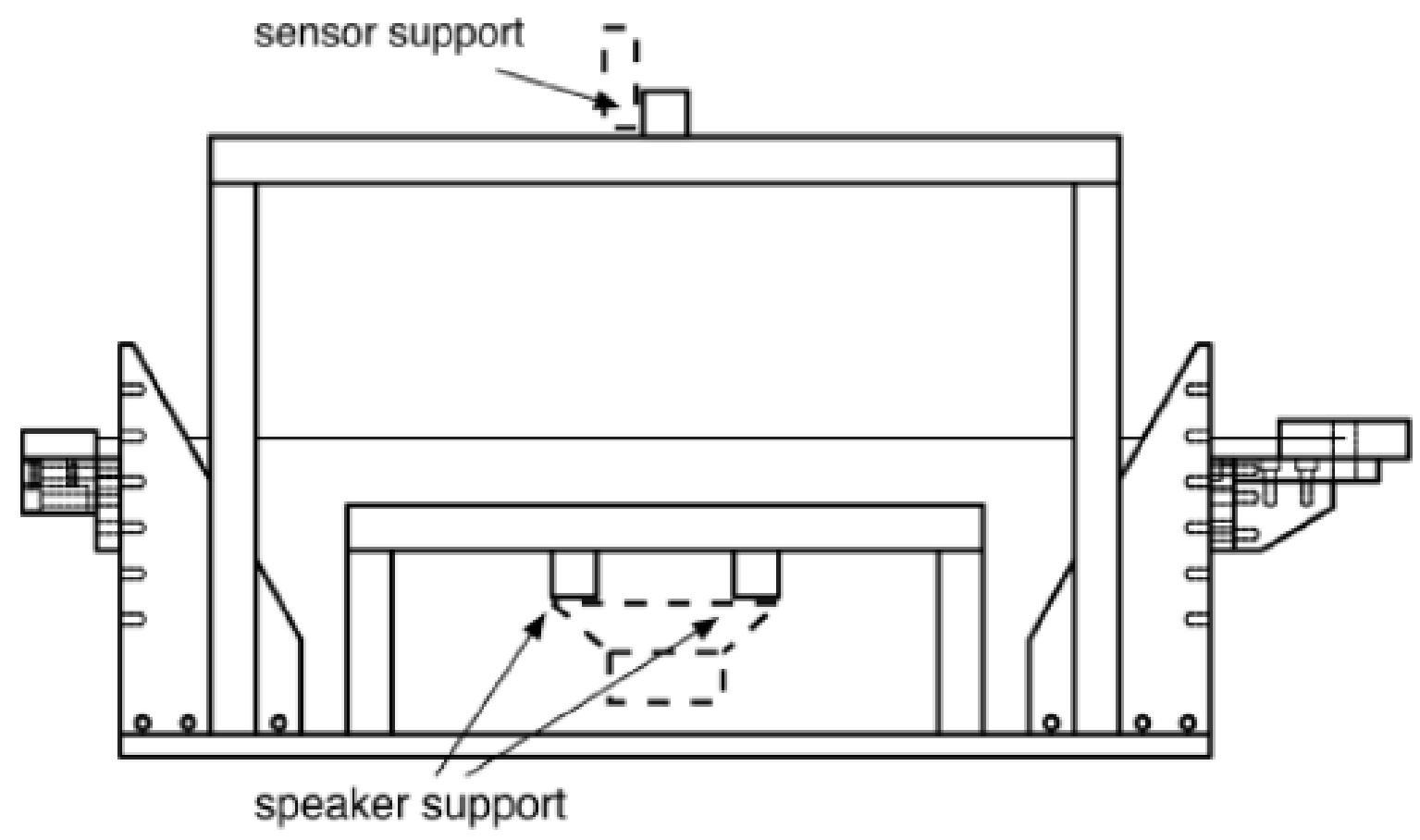

Figure 4 Side view of the "Mach Guitar" Mechanical Oscillator system

The restoring force provided by the tensioned wire pairs is $\mathbf{F}=-(\mathbf{8 T} / \mathbf{L}) \mathbf{x}$, where $T$ is the tension in a given wire, $\mathbf{L}$ is the overall length, from bridge to bridge, of the system, and $\mathbf{x}$ is the vertical displacement of the capacitor. Therefore, neglecting the mass of the wire, the resonant frequency of the oscillator is $\omega_{0}=(\mathbf{8 T} / \mathbf{m L})^{1 / 2}$. If $\mathbf{m}=1 \mathrm{~g}, \mathbf{L}=0.5 \mathrm{~m}$ and $\boldsymbol{\omega}_{0}=2 \pi \times 1000$ $\mathrm{Hz}$, then the required tension is $553 \mathrm{lb}$. This tension can be reached with 13 gauge steel wire.

The Mach Guitar is mounted on the laser-optical baseplate, which provides "bridges" to support for the wires and their tensioning mechanism. Electrical connections to the capacitance are made through the support wires. Below the oscillator is an audio speaker, which drives the oscillator through a light spring. Above the oscillator is a commercial laser position detector, which measures the vertical position of the capacitor's upper surface by electronic triangulation. The laser position sensor is shown in figure 5 .

The mass-varying object used in the measurements is a low-loss and low-mechanical-movement barium titanate capacitor with a capacitance of about $9 \mathrm{nF}$ and a voltage rating of $3 \mathrm{kV}$. This oscillator mass is suspended between pairs of 13 gauge steel wires $(0.25 \mathrm{~m}$ long on each side) that have been tensioned to about $500 \mathrm{lb}$ to provide a system resonant frequency of about $1000 \mathrm{~Hz}$.

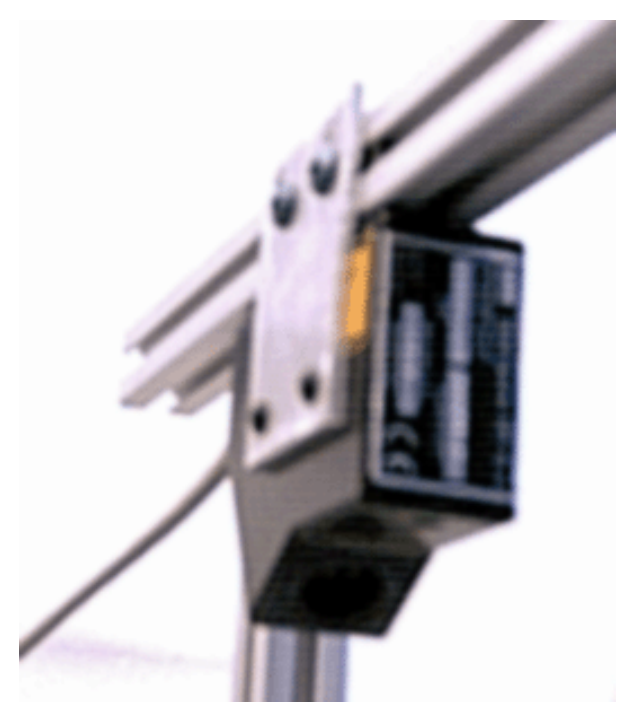

Figure 5 Laser position measurement device.

As previously mentioned, the most challenging problem presented by the present experiment is driving the capacitor to high voltages at audio frequencies. 
The reason is that all high-voltage amplifiers driving capacitive loads are severely limited by the charging current that they must deliver. We have selected a Trek Model PO923A High Voltage Power Amplifier, shown in figure 5, as the capacitor driver. It can drive at voltages up to $2 \mathrm{kV}$ with a peak charging current of up to $400 \mathrm{~mA}$.

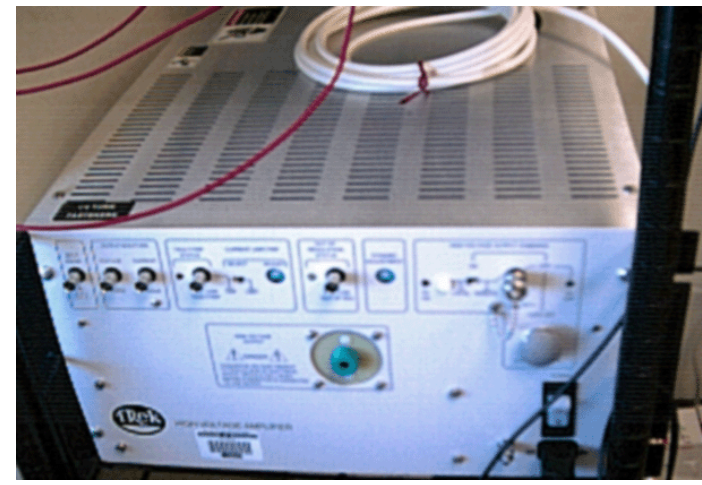

Figure 6 Trek Model PO923A HV Power Amplifier, used for driving the capacitor at $2 \mathrm{kV}$ and $400 \mathrm{~mA}$.

The Mach's Principle test employs a Pentium-2 $850 \mathrm{MHz}$ computer system with a Windows 98 operating system for experiment control, using control software based on LabView. It consists .of controls for the mechanical oscillator driver and the capacitance driver, and for the data collection system, as described above.

The system samples the mechanical drive voltage and the capacitor position measurement of the mechanical oscillator as separate data streams. These are sampled for real-time display and also recorded on the system hard disk. These data streams can be read back and re-analyzed. The data are analyzed by integration over a long time period to extract the relative phase of the drive and response signals for a given setting of the capacitor drive phase with respect to the mechanical driver.

The processed quantity that will be accumulated in the analysis is the cosine of the relative phase between the driving signal $\mathbf{D}(\mathbf{t})$ and the mechanical response signal $\mathbf{R}(\mathbf{t})$. There are a variety of ways of extracting this signal, but the one we will use initially is:

$\operatorname{Cos}(\varphi)=\frac{\int_{0}^{T}[D(t)+R(t)]^{2} d t-\int_{0}^{T}[D(t)-R(t)]^{2} d t}{4 \sqrt{\int_{0}^{T} D(t)^{2} d t \times \int_{0}^{T} R(t)^{2} d t}}$
Here $\mathbf{T}$ is an arbitrary integration time that increases as data is collected and the running integrals are accumulated. The values of $\operatorname{Cos}(\phi)$, which is near 0 because $\phi$ is approximately $\pi / 2$ on resonance, will be compared for the two most extreme settings of the phase of the capacitor drive, which should produce phase shifts like those shown in figure 2. We estimate that with a data collection cycle of a few hours, $\operatorname{Cos}(\phi)$ can be determined to an accuracy of a few parts in $10^{5}$. This should enable us to determine the shift in $\phi$ to similar accuracy, providing a fairly stringent test of the presence or absence of the Woodward mass variation.

\section{Theory of a Mass-Varying Driven Harmonic Oscillator}

We test for the presence of the Woodward effect by using the capacitor as the mass in a system that forms a driven mechanical mass-and-spring oscillator with an undriven resonant frequency of $\boldsymbol{\omega}_{\mathbf{0}}$. Such an oscillator is shown schematically in figure 7 .

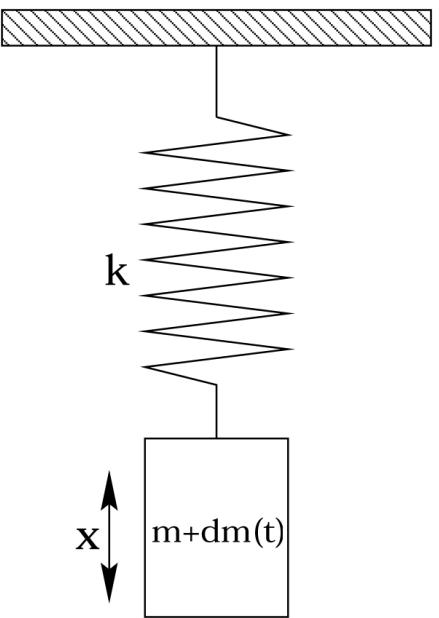

Figure 7 Schematic mass-and-spring mechanical oscillator with time varying mass $\mathbf{m}+\mathbf{d m}(\mathbf{t})$ and restoring-force spring constant $\mathbf{k}$. The system is assumed to have a dissipative damping force of $-b \mathbf{d x} / \mathbf{d t}$.

The oscillator is driven at its resonant frequency $\omega_{\mathrm{d}}=\left(\boldsymbol{\omega}_{\mathbf{0}}{ }^{2}-\mathbf{b} / \mathbf{2 m}\right)^{1 / 2}$ with a voice coil actuator and audio amplifier. At the same time, we charge the capacitor sinusoidally, using approximately the parameters specified above, at a frequency of $\omega_{d} / 2$ so that, in the presence of the Woodward effect, the capacitor's inertial mass should vary at frequency $\omega_{d}$. 
The inhomogeneous non-linear differential equation describing such a system is:

$$
\begin{aligned}
F_{d} \operatorname{Cos}\left[\omega_{d} t\right]=k x(t) & +\left[b+\mu^{\prime}(t)\right] x^{\prime}(t) \\
& +[m+\mu(t)] x^{\prime \prime}(t),
\end{aligned}
$$

where $\mathbf{x}(\mathbf{t})$ is the motion of the capacitor, $\mathbf{F}_{\mathbf{d}}$ is the magnitude of the driving force, $\boldsymbol{\omega}_{\mathbf{d}}$ is the angular frequency of the driving force, $\mathbf{k}$ is the Hooke's law restoring force constant, $\mathbf{b}$ is the damping constant representing dissipative forces in the system, $\mathbf{m}$ is the average mass of the capacitor and associated structure, and $\boldsymbol{\mu}(\mathbf{t})$ is the time-dependent mass variation due to the Woodward effect. Note that the $\mu^{\prime}(\mathbf{t}) \mathbf{x}^{\prime}(\mathbf{t})$ term in equation (6) arises from the $\mathbf{v ~} \mathbf{d m} / \mathbf{d t}$ term in equation (1).

We can replace the spring constant $\mathbf{k}$ with $\mathbf{m} \omega_{0}{ }^{2}$ and replace $\mu(\mathbf{t})$ with $\mu_{0} \operatorname{Cos}\left(\omega_{d} t+\phi_{m}\right)$, which assumes that we have arranged the mass variation to be at the same frequency as the driving force but shifted in phase by $\phi_{\mathbf{m}}$. With these substitutions, equation (6) becomes:

$$
\begin{aligned}
F_{d} \operatorname{Cos}\left(\omega_{d} t\right) & =m \omega_{0}{ }^{2} x(t) \\
+ & {\left[b-\mu_{0} \omega_{d} \operatorname{Sin}\left(\omega_{d} t+\varphi_{\mathrm{m}}\right)\right] x^{\prime}(t) } \\
+ & {\left[m+\mu_{0} \operatorname{Cos}\left(\omega_{d} t+\varphi_{\mathrm{m}}\right)\right] x^{\prime \prime}(t) }
\end{aligned}
$$

This non-linear differential equation has no analytic solutions and must be solved numerically. Figure 8 shows the results of such numerical solutions of equation (7), assuming that $\mathbf{F}_{\mathbf{d}} / \mathbf{m}=0.01, \mathbf{b} / \mathbf{m}=0.01$, and $\boldsymbol{\mu}_{\mathbf{0}} / \mathbf{m}=0.001$. The latter assumption represents only about $10 \%$ of the predicted $10 \mathrm{mg}$ mass variation.

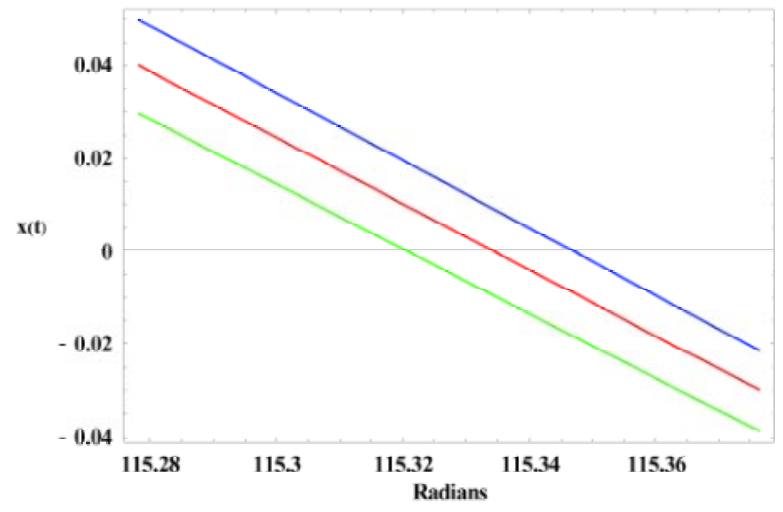

Figure 8. Response phase shifts of the system to variable mass. The central line is the system response with $\mu_{0}=0$. The other two lines represent $\mu_{0}=0.001$ with $\phi_{\mathrm{m}}=-\pi / 2$ (low), and $\phi_{\mathrm{m}}=+\pi / 2$ (high). The phase shifts shown are about \pm 0.04 radians $= \pm 2.3$ degrees.

We find that when the mass variation has a relative phase of $\pm \pi / 2$ with respect to the driving force, it causes a positive or negative phase shift in the response motion by shifts, using the values listed above, of several degrees. Other phases near 0 or $\pi$ can cause an increase or decrease in the amplitude of oscillation. The experiment we have constructed is designed in an attempt to observe these phase-shift effects.

\section{Experiment Status}

Test T1 described above was performed with the Mach Guitar arrangement, which looks for string oscillations when the predicted inertial mass variation (and presumably also gravitational mass variation) is set to match the resonant frequency of the mechanical system. Figure 9 shows the response of the system when the driving potential was applied to the capacitor.

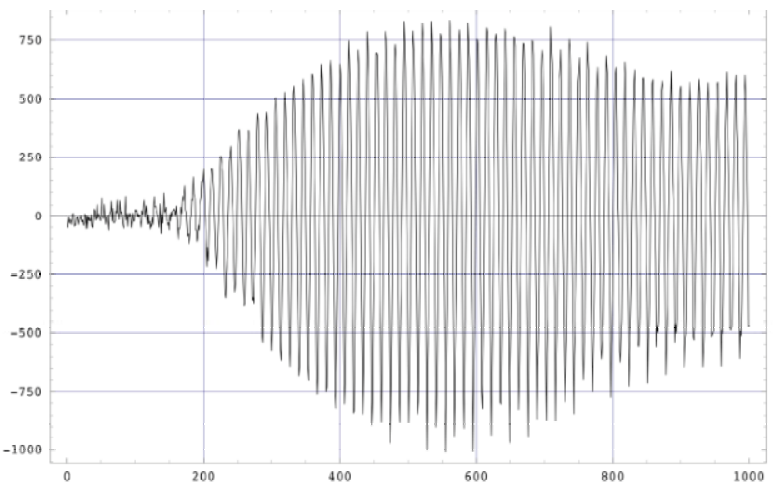

Figure 9. Position of the capacitor vs. time. Driving potential was applied at approximately $\mathrm{t}=180$, in arbitrary units.

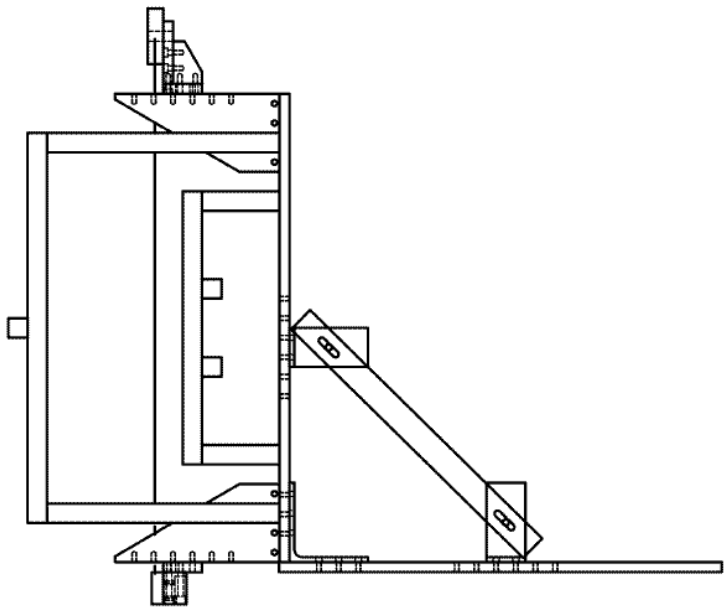

Figure 10. Consistency check: Mach Guitar apparatus rotated $90^{\circ}$, so that the tensioned strings are vertical and the predicted gravitational force is perpendicular to the allowed string displacement. 
In figure 9 we see that the position shows small variations before the charging voltage is applied at $\mathrm{t}=180$, and a rise to strong oscillations at the resonant frequency of the system after the charging voltage is applied. This result might be taken to be consistent with the predicted Woodward effect.

As a null check of this result, we rotated the Mach Guitar apparatus from a horizontal orientation to a vertical one, as shown in figure 10 . Now, if a varying gravitational force is present, it should be perpendicular to the allowed string displacement. Thus, we would expect only zero or small excitation of the oscillator. However, we find that in the $90^{\circ}$ orientation, the excitation of the mechanical oscillator is qualitatively the same as that shown in figure 9.

Since our null test failed, we conclude that it is not the Woodward Effect, but rather electrical forces induced by the large current flow to the capacitor that are producing the excitation seen in figure 9. The implication of this result is that there is no point in proceeding further with measurement $\mathrm{T} 1$ or performing measurement T2 until the spurious excitation shown in figure 9 is eliminated.

We are presently investigating ways of modifying the Mach Guitar apparatus so that the current drive is shielded or brought in using separate wires. When this problem is solved, we will continue with the T1 and T2 measurements.

\section{Conclusion and Summary}

The test of Mach's principle and the Woodward effect described above is not yet completed, but it shows promise of providing an independent test of the predictions that does not depend on the possibility of a reactionless force. The experiment in the present configuration is not as sensitive as the torsion-balance measurements recently reported by Woodward [7]. However, since it is not based on a reactionless force, it may not need that sensitivity.

If the Woodward Effect is observed, it will have important implications for general relativity and cosmology, for validating Mach's Principle, for control of inertia, and possibly for propulsion. If the
Woodward Effect is not observed at the sensitivity limit of the experiment, this will also be worth knowing.

The present configuration of the apparatus has a background that spontaneously excites the mechanical oscillator when the capacitor is driven. This effect must be eliminated before the measurements can proceed further. Work toward this end is currently in progress.

\section{Bibliography}

[1] D. Sciama, "On the Origin of Inertia," Monthly Notices of the Royal Astronomical Society 113, 34-42 (1953).

[2] D. Sciama, "The Physical Structure of General Relativity," Reviews of Modern Physics 36, 463-469 (1964).

[3] Woodward, J.F., "A New Experimental Approach to Mach's Principle and Relativistic Gravitation," Foundations of Physics Letters 3, 497-506 (1990).

[4] James F. Woodward, "Measurements of a Machian Transient Mass Fluctuation," Foundations of Physics Letters 4, 407-423 (1991).

[5] James F. Woodward ,"A Stationary Apparent Weight Shift from a Transient Machian Mass Fluctuation," Foundations of Physics Letters 5, 425-442 (1992).

[6] James F. Woodward, “A Laboratory Test of Mach's Principle and Strong-Field Relativistic Gravity," Foundations of Physics Letters 9, 247-293 (1996).

[7] James F. Woodward, "Mass Fluctuations, Stationary Forces, and Propellantless Propulsion," Space Technology and Applications International Forum 2000 (American Institute of Physics/Springer Verlag, New York, 2000), 1018-1025 (2000). 


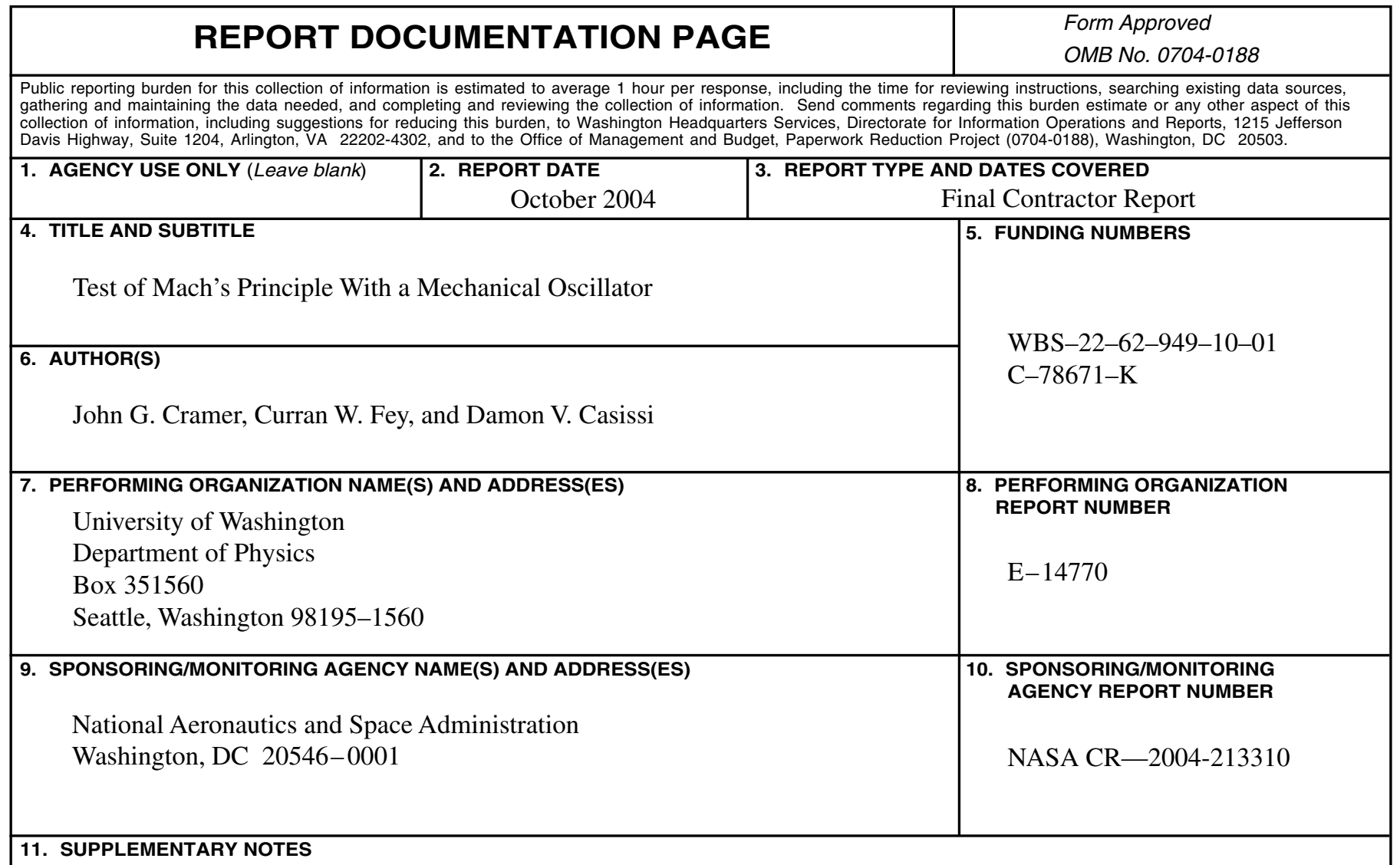

Project Manager, Marc G. Millis, Turbomachinery and Propulsion Systems Division, NASA Glenn Research Center, organization code 5870, 216-977-7535.

12a. DISTRIBUTION/AVAILABILITY STATEMENT

12b. DISTRIBUTION CODE

Unclassified - Unlimited

Subject Categories: 20 and 70

Distribution: Nonstandard

Available electronically at http://gltrs.grc.nasa.gov

This publication is available from the NASA Center for AeroSpace Information, 301-621-0390.

13. ABSTRACT (Maximum 200 words)

James F. Woodward has made a prediction, based on Sciama's formulation of Mach's Principle in the framework of general relativity, that in the presence of an energy flow the inertial mass of an object may undergo sizable variations, changing as the second time derivative of the energy. We describe an attempt to test for the predicted effect with a charging capacitor, using a technique that does not require an unbalanced force or any local violation of Newton's 3rd law of motion. We attempt to observe (1) the gravitational effect of the varying mass and (2) the effect of the mass variation on a driven harmonic oscillator with the charging capacitor as the oscillating mass. We report on the predicted effect, the design and implementation of the measurement apparatus, and initial experience with the apparatus. At this time, however, we will not report on observations of the presence or absence of the Woodward effect.

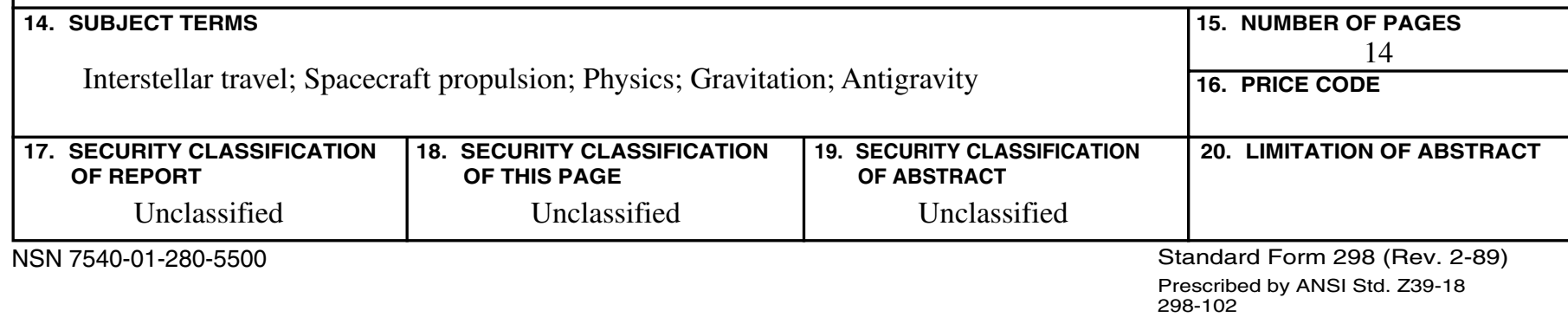



